\title{
Deglutição e nutrição em pacientes com câncer de orofaringe: Uma revisão
}

\author{
sistemática \\ Deglutition and nutrition in patients with oropharyngeal cancer: A systematic review \\ Deglución y nutrición en pacientes con cáncer de orofaringe: Una revisión sistemática
}

Recebido: 28/06/2021 | Revisado: 04/07/2021 | Aceito: 08/07/2021 | Publicado: 21/07/2021

Júlia Ligocki Pinto Weschenfelder

ORCID: https://orcid.org/0000-0003-0467-7660

Universidade Federal de Santa Catarina, Brasil E-mail: julialpwesch@gmail.com

Laura Faustino Gonçalves

ORCID: https://orcid.org/0000-0002-0043-4349 Universidade Federal de Santa Catarina, Brasil E-mail: laurafaustinog@outlook.com

Cláudia Tiemi Mituuti

ORCID: https://orcid.org/0000-0002-8991-3812 Universidade Federal de Santa Catarina, Brasil E-mail: claudia.mituuti@ufsc.br

Patrícia Haas

ORCID: https://orcid.org/0000-0001-9797-7755

Universidade Federal de Santa Catarina, Brasil

E-mail: patricia.haas@ufsc.br

\begin{abstract}
Resumo
O objetivo do presente estudo é apresentar evidências científicas com base em revisão sistemática da literatura (PRISMA) respondendo à pergunta norteadora: A deglutição e aspectos nutricionais são afetados em pacientes com câncer de orofaringe? A busca de artigos foi realizada nas bases de dados Scielo, Lilacs, Pubmed, Scopus, Bireme e Web Of Science, não houve restrição de localização, período e idioma. Para a seleção dos estudos foi utilizada a combinação baseada no Medical Subject Heading Terms (MeSH). Para complementar, foi realizada uma busca por literatura cinza no Google Scholar. Foram identificados 132 artigos com potencial para inclusão, sendo que 3 corresponderam aos critérios de inclusão definidos e à pergunta norteadora. Pacientes com câncer de cabeça e pescoço, que apresentaram queixa de disfagia tiveram a eficiência de deglutição diminuída, além do trânsito mais longo, presença de resíduo e aspiração, em comparação aos que não apresentaram a queixa. O ganho nutricional via enteral, em alguns casos, não é suficiente, necessitando de alternativas, como a hiperalimentação venosa. A capacidade de perceber a função da deglutição pode ser útil para automonitoramento de mudanças no estado da disfagia durante terapia de deglutição. Adicionar terapia de deglutição não melhora a normalidade de ingestão oral, mas acelera a recuperação da deglutição de alimentos. A hiperalimentação venosa é uma alternativa viável e eficiente em alguns casos, para suprir necessidades nutricionais dos pacientes com câncer em que não há possibilidade de alimentação por via oral e diante de problemas que podem impedir a nutrição via enteral.
\end{abstract}

Palavras-chave: Deglutição; Transtornos da deglutição; Neoplasias orofaríngeas; Orofaringe.

\begin{abstract}
The aim of this study is to present scientific evidence based on a systematic literature review (PRISMA) answering the guiding question: Are swallowing and nutritional aspects affected in patients with oropharyngeal cancer? The search for articles was carried out in the databases Scielo, Lilacs, Pubmed, Scopus, Bireme and Web Of Science, there was no restriction on location, period and language. For the selection of studies, the combination based on the Medical Subject Heading Terms (MeSH) was used. In addition, a search for gray literature was carried out on Google Scholar. 132 articles with potential for inclusion were identified, 3 of which met the defined inclusion criteria and the guiding question. Patients with head and neck cancer, who presented complaints of dysphagia had decreased swallowing efficiency, in addition to longer transit, presence of residue and aspiration, compared to those who did not complain. The nutritional gain via enteral, in some cases, is not enough, requiring alternatives, such as venous hyperalimentation. The ability to perceive the function of swallowing can be useful for self-monitoring changes in the state of dysphagia during swallowing therapy. Adding swallowing therapy does not improve normal food intake but accelerates the recovery of swallowing function. Venous hyperalimentation is a viable and efficient alternative in some cases, to meet
\end{abstract}


the nutritional needs of cancer patients in which there the oral feeding is not possible and in the face of problems with enteral nutrition.

Keywords: Deglutition; Deglutition disorders; Oropharyngeal neoplasms; Oropharynx.

\section{Resumen}

El propósito de este estudio es presentar evidencia científica basada en una revisión sistemática de la literatura (PRISMA) que responda a la pregunta orientadora: ¿Se ven afectados la deglución y los aspectos nutricionales en pacientes con cáncer de orofaringe? La búsqueda de artículos se realizó en las bases de datos Scielo, Lilacs, Pubmed, Scopus, Bireme y Web Of Science, no hubo restricción de ubicación, época e idioma. Para la selección de estudios se utilizó la combinación basada en los Términos de Encabezamiento de Materia Médica (MeSH). Además, se realizó una búsqueda de literatura gris en Google Scholar. Se identificaron 132 artículos con potencial de inclusión, 3 de los cuales cumplieron con los criterios de inclusión definidos y la pregunta orientadora. Los pacientes con cáncer de cabeza y cuello, que se quejaron de disfagia, presentaron disminución de la eficiencia de la deglución, además de mayor tránsito, presencia de residuo y aspiración, en comparación con los que no se quejaron. La ganancia nutricional vía enteral, en algunos casos, no es suficiente, requiriendo alternativas, como la hiperalimentación venosa. La capacidad de percibir la función de la deglución puede ser útil para el autocontrol de los cambios en el estado de disfagia durante la terapia de deglución. Agregar terapia de deglución no mejora la ingesta oral, pero acelera la recuperación de la deglución. La hiperalimentación venosa es una alternativa viable y eficaz en algunos casos, para cubrir las necesidades nutricionales de los pacientes oncológicos en los que no es posible la alimentación oral y ante problemas de nutrición enteral.

Palabras clave: Degluticíon; Transtornos de degluticíon; Neoplasias orofaríngeas; Orofaringe.

\section{Introdução}

O câncer é uma doença com várias causas, como os fatores ambientais, culturais, socioeconômicos, estilos de vida ou costumes, os hábitos alimentares, fatores genéticos, própria ação de envelhecimento e o ato de fumar (Oliveira, et al., 2015). O câncer de cabeça e pescoço (CCP) possui uma grande incidência de mortes no mundo, sendo, em média, a terceira causa mais corriqueira de morte por câncer. Pode acometer a cavidade oral, orofaringe, hipofaringe, nasofaringe e laringe (Galbiatti, et al., 2013).

A alimentação é essencial para manter os indivíduos nutridos e hidratados e nesse processo de levar o alimento da boca até o estômago, acontece a deglutição. É considerado um recurso neuromuscular complexo, que abrange diversos aspectos, e envolve estruturas relacionadas à cavidade oral, laringe, faringe e esôfago. A disfagia, caracterizada como distúrbio no processo de deglutição, é um sintoma de uma doença de base, que pode acometer qualquer parte do trato digestivo e acarretar em algumas complicações, como desnutrição, desidratação e problemas respiratórios, como pneumonia (Furkim \& Sacco, 2008).

No Brasil, o CCP é muito recorrente, onde o tratamento é complexo, multidisciplinar e multimodal. A causa, muitas vezes, está associada ao tabagismo e etilismo e acomete, predominantemente, o sexo masculino e indivíduos com mais de 50 anos (Dedivitis, et al., 2004). De uma forma geral, os indivíduos com CCP podem apresentar dificuldade para se comunicar, se alimentar e podem apresentar perda de peso, em decorrência da doença e dos tratamentos. A disfagia e alterações vocais também podem ocorrer, em consequência destes tratamentos, afetando o indivíduo (Aquino, et al., 2016).

O estado nutricional de pacientes com CCP pode estar comprometido, em decorrência das alterações metabólicas relacionadas ao tumor, às dificuldades para se alimentar em decorrência de diversos fatores, e à caquexia associada ao câncer. A localização e o tratamento do câncer, nessa região de cabeça e pescoço, também podem prejudicar seriamente o estado nutricional do paciente, levando a impactos negativos na qualidade de vida (QV), morbidade, mortalidade, além de afetar aspectos físicos e emocionais (Oliveira, et al., 2015).

A partir do exposto, a presente pesquisa apresenta como objetivo principal e norteador verificar evidências científicas se a deglutição e aspectos nutricionais são afetadas em pacientes com câncer de orofaringe, visando responder a seguinte 
pergunta norteadora de pesquisa: A deglutição e aspectos nutricionais são afetados em pacientes com câncer de orofaringe?

\section{Metodologia}

\section{Protocolo e registro}

A presente revisão sistemática foi conduzida conforme as recomendações PRISMA (Preferred Reporting Items for Systematic reviews and Meta-Analyses) (Shamseer, et al., 2015).

As buscas por artigos científicos foram conduzidas por dois pesquisadores independentes nas bases de dados eletrônicas MEDLINE (Pubmed), LILACS, SciELO, SCOPUS, WEB OF SCIENCE e BIREME, sem restrição de idioma, período e localização. A pesquisa foi estruturada e organizada na forma PICOS, que representa um acrônimo para População alvo, a Intervenção, Comparação e “Outcomes” (desfechos). População de interesse ou problema de saúde (P) corresponde à pacientes; intervenção (I): câncer de orofaringe; comparação $(\mathrm{C})$, aspectos; outcome $(\mathrm{O})$ : deglutição e nutrição; (S): estudo transversal, estudo observacional, relatos de caso, estudos de caso-controle, ensaios clínicos controlados, estudos de coorte.

Tabela 1. Descrição dos componentes do PICOS.

\begin{tabular}{|l|l|}
\hline Acrônimo & Definição \\
\hline P & Pacientes \\
\hline I & Câncer de Orofaringe \\
\hline C & Aspectos \\
\hline O & Deglutição nutrição \\
\hline S & $\begin{array}{l}\text { Estudo transversal } \\
\text { Estudo observacional } \\
\text { Relatos de caso } \\
\text { Estudos de caso-controle } \\
\text { Ensaios clínicos controlados } \\
\end{array}$ \\
\hline
\end{tabular}

Fonte: Autores.

\section{Estratégia de pesquisa}

Os descritores foram selecionados a partir do dicionário Descritores em Ciências da Saúde (DeCS) e Medical Subject Heading Terms (MeSH), haja vista a sua grande utilização pela comunidade científica para a indexação de artigos na base de dados PubMed. Diante da busca dos descritores, foi realizada a adequação para as outras bases utilizadas. Em um primeiro momento foram propostas para as buscas os seguintes descritores e operadores booleanos: (Swallowing) and (Head and Neck Neoplasms) or (Oropharynx) and (Food) and (nutrition). A busca ocorreu em novembro de 2020. Para complementar, foi realizada uma busca por literatura cinza no Google Scholar.

\section{Critérios de Elegibilidade}

Os desenhos do estudo selecionado poderiam ser do tipo descritivo, transversal, observacional, de caso-controle, de coorte, ensaios clínicos controlados, relatos de caso estudo e estudo qualitativo. Foram inclusos estudos sem restrição de idioma, período e localização. A Tabela 2 representa os critérios de inclusão e exclusão desenvolvidos nesta pesquisa. Os 
Research, Society and Development, v. 10, n. 9, e0310917630, 2021

(CC BY 4.0) | ISSN 2525-3409 | DOI: http://dx.doi.org/10.33448/rsd-v10i9.17630

estudos obtiveram pontuação 12 no protocolo modificado de Pithon et al. (2015) para avaliação da qualidade dos mesmos.

Tabela 2. Síntese dos critérios de inclusão/exclusão.

\begin{tabular}{ll}
\hline Critérios de Inclusão & Relatos de casos \\
\hline Delineamento & Estudos de caso-controle \\
& Ensaios clínicos controlados \\
& Estudos de coorte \\
& Estudos em triagem \\
Estudos observacionais & Sem restrição \\
Ldioma & Sem restrição \\
\hline Critérios de Exclusão & Cartas ao editor \\
\hline Delineamento & Diretrizes \\
& Revisões de literatura \\
Forma de publicação & Revisões sistemáticas \\
\hline & Meta-análises \\
Estudos & Estudos pouco claros \\
& Mal descritos ou inadequados \\
& Apenas resumo \\
\hline
\end{tabular}

Fonte: Autores.

\section{Risco de viés}

A qualidade dos métodos foi avaliada pelos revisores de forma independente (CTM e PH), de acordo com a recomendação PRISMA (Shamseer, et al., 2015). A avaliação priorizou a descrição clara das informações. Neste ponto, a revisão foi realizada às cegas, mascarando os nomes dos autores e revistas, evitando qualquer viés potencial e conflito de interesses.

\section{Critérios de Exclusão}

Foram excluídos estudos publicados nos formatos de Cartas ao editor, diretrizes, revisões de literatura, revisões narrativas, revisões sistemáticas, meta análises e resumos. Estudos indisponíveis na íntegra, também foram excluídos (Tabela 2).

\section{Análise dos dados}

A extração dos dados para o processo de elegibilidade dos estudos foi realizada utilizando-se uma ficha própria para revisão sistemática elaborada por dois pesquisadores em Programa Excel®, na qual os dados extraídos foram adicionados por um dos pesquisadores e, então, conferidos por outro pesquisador. Inicialmente foram selecionados de acordo com o título; em seguida, os resumos foram analisados e apenas os que fossem potencialmente elegíveis foram selecionados. Com base nos 
Research, Society and Development, v. 10, n. 9, e0310917630, 2021

(CC BY 4.0) | ISSN 2525-3409 | DOI: http://dx.doi.org/10.33448/rsd-v10i9.17630

resumos, artigos foram selecionados para leitura integral, foram admitidos os que atendiam a todos os critérios prédeterminados. Em caso de desacordo entre avaliadores, um terceiro avaliador (KMP) tomou a decisão sobre a elegibilidade do estudo em questão.

\section{Forma de seleção dos estudos}

Inicialmente os revisores de elegibilidade (CTM e PH) foram calibrados para a realização da revisão sistemática por JLPW e LFG. Após a calibração e esclarecimentos de dúvidas, os títulos e resumos foram examinados por dois revisores de elegibilidade (CTM e PH), de forma independente, os quais não estavam cegos para o nome dos autores e das revistas. Aqueles que apresentaram um título dentro do âmbito, mas os resumos não estavam disponíveis, também foram obtidos e analisados na íntegra. Foram excluídos estudos fora do escopo proposto, relatos de caso, cartas ao editor e/ou editorial, revisões de literatura, índices, resumos e estudos em animais. Posteriormente, os estudos elegíveis preliminarmente tiveram o texto completo obtido e avaliado. Em casos específicos, quando o estudo com potencial de elegibilidade apresentasse dados incompletos, os autores poderiam ser contatados por e-mail para mais informações, no entanto não foi necessário este contato.

\section{Dados Coletados}

Após a triagem, o texto do artigo selecionado foi revisado e extraído de forma padronizada por dois autores (JLPW e LFG) sob a supervisão de CTM e PH, identificando-se ano de publicação, local da pesquisa, idioma de publicação, tipo de estudo, amostra, método, resultado e conclusão do estudo.

\section{Resultado clínico}

O resultado clínico de interesse foi verificar evidências científicas se a deglutição e nutrição são afetados em pacientes com câncer de orofaringe. Aqueles que não utilizaram esta abordagem específica não fizeram parte da amostra admitida para a revisão de literatura.

\section{Resultados e Discussão}

Inicialmente foram selecionados 132 artigos, dimensionados para 122 após exclusão por repetição; em seguida, os títulos e resumos foram analisados e 118 trabalhos foram excluídos pois não estavam no escopo da proposta da pesquisa. Assim, admitiram-se 3 artigos (Sobol, et al., 1979; Pauloski, et al., 2002; Berg, et al., 2015) para a análise final, sendo eles do tipo estudo de caso (Figura 1). 
Research, Society and Development, v. 10, n. 9, e0310917630, 2021

(CC BY 4.0) | ISSN 2525-3409 | DOI: http://dx.doi.org/10.33448/rsd-v10i9.17630

Figura 1. Fluxograma de busca e análise dos artigos.

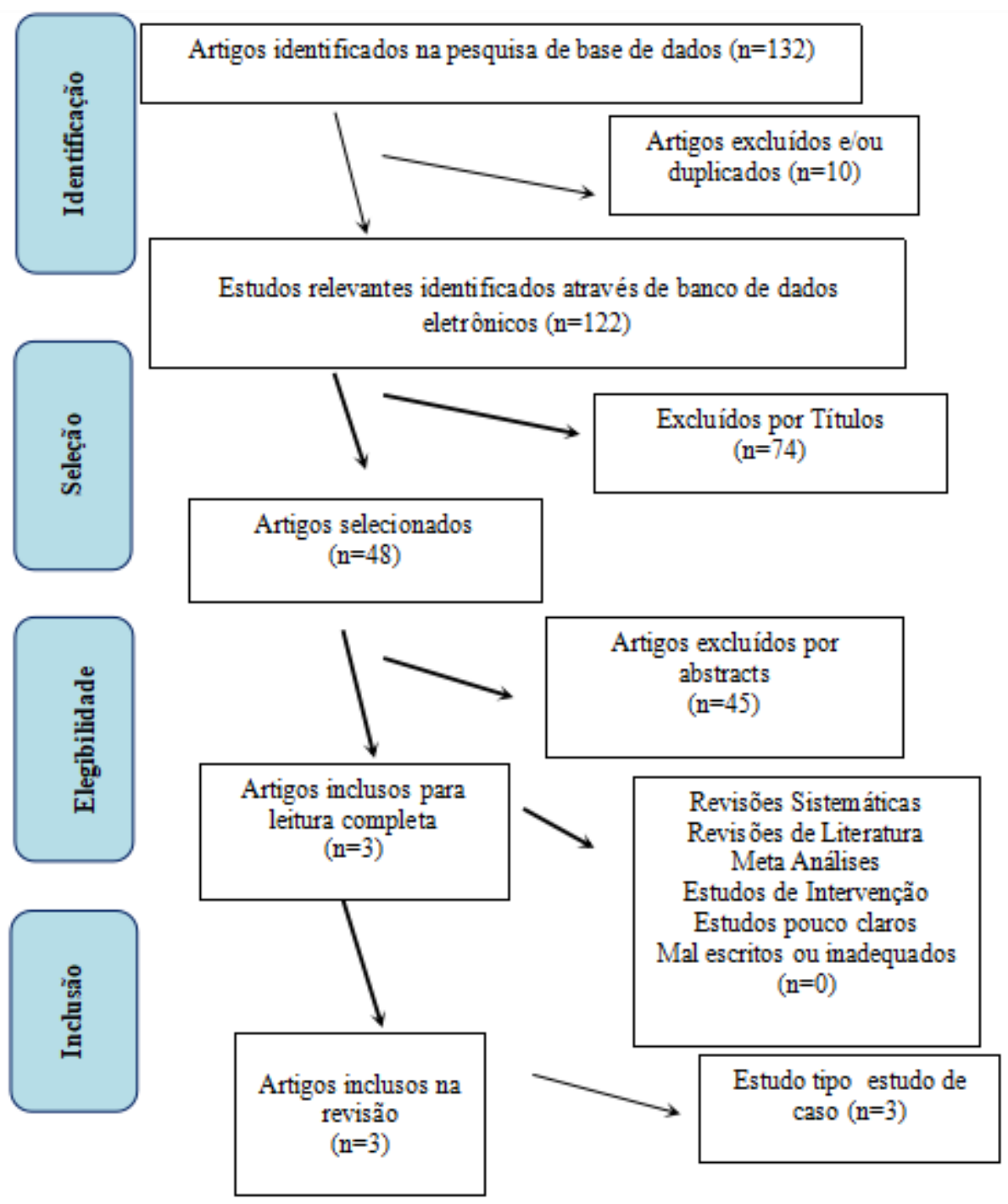

Fonte: Autores.

A partir dos descritores eleitos, os bancos de dados foram consultados e foram obtidos os resultados disponibilizados na Tabela 3 . 
Research, Society and Development, v. 10, n. 9, e0310917630, 2021

(CC BY 4.0) | ISSN 2525-3409 | DOI: http://dx.doi.org/10.33448/rsd-v10i9.17630

Tabela 3. Classificação das referências obtidas nas base de dados Pubmed, Scielo, Lilacs, Web Of Science e Scopus.

\begin{tabular}{|c|c|c|c|c|c|}
\hline Descritores & $\mathbf{N}^{\mathbf{o}}$ & $\begin{array}{c}\text { Referências } \\
\text { excluídas }\end{array}$ & Motivo & Selecionado & Banco de dados \\
\hline $\begin{array}{l}\text { (Swallowing) } \\
\text { and (Head and } \\
\text { Neck } \\
\text { Neoplasms) or } \\
\text { (Oropharynx) } \\
\text { and (Food) and } \\
\text { (nutrition). }\end{array}$ & 117 & 114 & $\begin{array}{l}\text { Duplicados (10); } \\
\text { excluídos por } \\
\text { título (67); } \\
\text { excluídos por } \\
\text { resumo (37) }\end{array}$ & 3 & Pubmed \\
\hline $\begin{array}{l}\text { (Swallowing) } \\
\text { and (Head and } \\
\text { Neck } \\
\text { Neoplasms) or } \\
\text { (Oropharynx) } \\
\text { and (Food) and } \\
\text { (nutrition). }\end{array}$ & 0 & - & - & - & Lilacs \\
\hline $\begin{array}{l}\text { (Swallowing) } \\
\text { and (Head and } \\
\text { Neck } \\
\text { Neoplasms) or } \\
\text { (Oropharynx) } \\
\text { and (Food) and } \\
\text { (nutrition). }\end{array}$ & 0 & - & - & - & Scielo \\
\hline $\begin{array}{l}\text { (Swallowing) } \\
\text { and (Head and } \\
\text { Neck } \\
\text { Neoplasms) or } \\
\text { (Oropharynx) } \\
\text { and (Food) and } \\
\text { (nutrition). }\end{array}$ & 0 & - & - & - & $\begin{array}{l}\text { WEB OF } \\
\text { SCIENCE }\end{array}$ \\
\hline $\begin{array}{l}\text { (Swallowing) } \\
\text { and (Head and } \\
\text { Neck } \\
\text { Neoplasms) or } \\
\text { (Oropharynx) } \\
\text { and (Food) and } \\
\text { (nutrition). }\end{array}$ & 15 & 15 & $\begin{array}{l}\text { Excluídos por } \\
\text { título (7); } \\
\text { excluídos por } \\
\text { resumo (8) }\end{array}$ & 0 & Bireme \\
\hline $\begin{array}{l}\text { (Swallowing) } \\
\text { and (Head and } \\
\text { Neck } \\
\text { Neoplasms) or } \\
\text { (Oropharynx) } \\
\text { and (Food) and } \\
\text { (nutrition). }\end{array}$ & 0 & - & & - & SCOPUS \\
\hline Total & 132 & 129 & & 3 & Pubmed \\
\hline
\end{tabular}

Fonte: Autores.

Com relação a descrição dos resultados dos artigos elegíveis neste estudo, as informações podem ser verificadas detalhadamente na 
Tabela 4. Os métodos utilizados, bem como resultados e conclusão das pesquisas se encontram expostos na referida tabela.

Tabela 4. Síntese dos artigos incluídos.

\begin{tabular}{|c|c|c|c|c|c|}
\hline $\begin{array}{c}\text { Autor / Ano / } \\
\text { Local de } \\
\text { publicação }\end{array}$ & Objetivo & $\mathbf{N}$ da amostra & Método & Resultados & Conclusão \\
\hline $\begin{array}{l}\text { Sobol et al., } \\
1979\end{array}$ & $\begin{array}{l}\text { Discute o caso de dois } \\
\text { pacientes com câncer } \\
\text { de } \\
\text { cabeça e pescoço, que } \\
\text { aborda sobre a } \\
\text { nutrição e a } \\
\text { necessidade de } \\
\text { escolha do método de } \\
\text { nutrição adequado. }\end{array}$ & 2 & $\begin{array}{l}\text { Relato de casos de } \\
\text { dois pacientes que } \\
\text { Apresentavam câncer } \\
\text { de cabeça e pescoço, } \\
\text { discutindo o estado } \\
\text { nutricional } \\
\text { alternativas para o } \\
\text { suporte nutricional. }\end{array}$ & $\begin{array}{l}\text { Quando a alimentação } \\
\text { enteral não supre a } \\
\text { necessidade dos } \\
\text { pacientes com câncer, } \\
\text { então a } \\
\text { hiperalimentação } \\
\text { intravenosa é utilizada } \\
\text { para restaurar o estado } \\
\text { nutricional dos } \\
\text { pacientes. }\end{array}$ & $\begin{array}{l}\text { A estratégia de reabilitação } \\
\text { nutricional precisa ser } \\
\text { planejada e acompanhada. } \\
\text { A alimentação enteral } \\
\text { muitas vezes não atende a } \\
\text { demanda dos pacientes } \\
\text { com câncer, então a } \\
\text { hiperalimentação venosa é } \\
\text { uma alternativa viável e } \\
\text { eficiente em alguns casos. }\end{array}$ \\
\hline $\begin{array}{l}\text { Pauloski et } \\
\text { al., } 2002\end{array}$ & $\begin{array}{l}\text { Analisar a relação da } \\
\text { queixa de disfagia e } \\
\text { função da deglutição, } \\
\text { em pacientes com } \\
\text { câncer de cabeça e } \\
\text { pescoço. }\end{array}$ & 132 & $\begin{array}{l}\text { A deglutição foi } \\
\text { avaliada por meio da } \\
\text { videofluoroscopia e as } \\
\text { queixas de disfagia } \\
\text { dos pacientes foram } \\
\text { analisadas. }\end{array}$ & 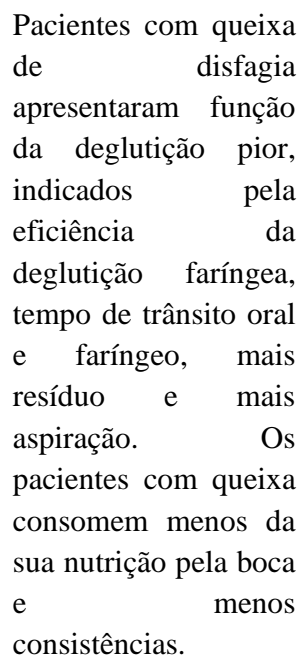 & $\begin{array}{l}\text { Os pacientes perceberam } \\
\text { decréscimos das suas } \\
\text { funções da deglutição e, } \\
\text { consequentemente, podem } \\
\text { ter limitado sua ingestão } \\
\text { oral. A capacidade de } \\
\text { perceber sua função da } \\
\text { deglutição pode ser útil } \\
\text { para automonitoramento de } \\
\text { mudanças no estado da } \\
\text { disfagia durante terapia de } \\
\text { deglutição. }\end{array}$ \\
\hline $\begin{array}{l}\text { Berg et al., } \\
2015 \\
\text { Holanda }\end{array}$ & $\begin{array}{l}\text { Investigar o efeito do } \\
\text { aconselhamento da } \\
\text { dieta } \\
\text { individual combinada } \\
\text { com terapia de } \\
\text { deglutição } \\
\text { individualizada, em } \\
\text { comparação com a } \\
\text { dieta } \\
\text { individual sobre a } \\
\text { normalidade de } \\
\text { ingestão } \\
\text { alimentar. }\end{array}$ & 120 & $\begin{array}{l}\text { Pacientes com câncer } \\
\text { de cabeça e pescoço, } \\
\text { tratados } \\
\text { radioterapia } \\
\text { quimioterapia pós- } \\
\text { operatória, obtiveram } \\
\text { medidas de gravidade } \\
\text { de disfagia, } \\
\text { normalidade da } \\
\text { ingestão oral, } \\
\text { alimentação social e } \\
\text { estado nutricional ao } \\
\text { longo de } 30 \text { semanas. }\end{array}$ & $\begin{array}{l}\text { Não foram detectadas } \\
\text { diferenças entre os } \\
\text { grupos para o efeito } \\
\text { da dieta individual, } \\
\text { terapia de deglutição } \\
\text { individualizada, } \\
\text { alimentação social e } \\
\text { normalidade de } \\
\text { ingestão alimentar. } \\
\text { Apenas uma ligeira } \\
\text { melhora ao longo das } \\
\text { semanas, mas que, no } \\
\text { final, a diferença entre } \\
\text { os grupos diminuiu } \\
\text { novamente. }\end{array}$ & $\begin{array}{l}\text { Adicionar terapia de } \\
\text { deglutição individualizada } \\
\text { ao aconselhamento } \\
\text { dietético individual não } \\
\text { melhora a normalidade da } \\
\text { ingestão de alimentos, mas } \\
\text { acelera ligeiramente a } \\
\text { recuperação da deglutição. }\end{array}$ \\
\hline
\end{tabular}

Fonte: Pauloski et al. (2002); Sobol et al., (1979); Pauloski et al., (2002); Berg et al., (2015).

\section{Desenho dos estudos}

No estudo feito por Berg, et al. (2015) foram selecionados pacientes com câncer de cabeça e pescoço em estágio II-IV, 
Research, Society and Development, v. 10, n. 9, e0310917630, 2021

(CC BY 4.0) | ISSN 2525-3409 | DOI: http://dx.doi.org/10.33448/rsd-v10i9.17630

onde tiveram carcinoma de células escamosas na cavidade oral, nasofaringe, orofaringe, hipofaringe ou laringe em tratamento com (quimio) radioterapia. Foram medidos a gravidade da disfagia, alimentação social, estado nutricional e a normalidade da ingestão alimentar no início do tratamento e ao longo das semanas. Além disso, investigaram o efeito sobre a ingestão alimentar do aconselhamento da dieta individual combinado com terapia de deglutição individualizada em comparação com o aconselhamento da dieta individual sozinho. Foram selecionados 57 pacientes em cada grupo para análise.

No segundo estudo de Sobol, et al. (1979) são apresentados dois casos de pacientes com CCP, um avaliado por meio de videofluoroscopia da deglutição e outro com queixas de disfagia e odinofagia decorrente da mucosite, ressaltando a necessidade de escolher o método ideal para suplementação nutricional, evitando a desnutrição.

Na pesquisa de Pauloski, et al. (2002), foram analisados a função da deglutição de cento e trinta e dois pacientes com câncer de cavidade oral, faringe e laringe, após o tratamento com radioterapia e quimioterapia. A função era avaliada por meio da videofluoroscopia da deglutição, além disso, os pacientes informavam quais consistências e quantidade de alimentos que ingeriam.

\section{Eficiência da deglutição e nutrição}

Para o grupo de intervenção no estudo de Berg, et al. (2015), 14\% das medidas de capacidade de deglutição não foram realizadas no grupo de intervenção e $19 \%$ no grupo controle, uma vez que os pacientes não foram capazes de engolir, também em consequência da dor e aspiração. A porcentagem de pacientes que apresentaram a pontuação de normalidade de ingestão de alimentos ideal na semana 30 foi quase igual a da semana 0 . A porcentagem de pacientes com pontuações ideais na gravidade de disfagia e alimentação social, em ambos os grupos, foram semelhantes na semana 0 e 30 (Berg, et al., 2015).

O segundo estudo (Sobol, et al. 1979) relata um caso de uma massa cervical esquerda, que sofreu com esgotamento nutricional gradual e progressivo devido ao câncer e dificuldade de manejo da nutrição pela extensa cirurgia, recontrução e necrose do tecido. O segundo relata sobre um caso de câncer que envolve a região das pregas vocais e epiglote, de um paciente com história de cigarro e abuso de álcool, que obteve um ganho nutricional insuficiente devido a severidade da mucosite oral decorrente da radioterapia e intolerância da alimentação por via enteral, necessitando de hiperalimentação intravenosa.

No estudo de Pauloski, et al. (2002) foi observado a eficiência da deglutição orofaríngea, a partir das medidas da deglutição e observações, que incluíam tempo de trânsito oral, faríngeo e resíduos, com doses de $1 \mathrm{ml}, 3 \mathrm{ml}, 5 \mathrm{ml}$ e $10 \mathrm{ml}$ de bário e pasta de bário com pudim de chocolate. Pacientes com queixa de disfagia tiveram a eficiência de deglutição diminuída, além do trânsito oral e faríngeo mais longo, em comparação aos que não apresentam queixa. Pacientes com queixa apresentaram resíduo oral e faríngeo significantemente maior do que pacientes sem queixa de deglutição e também apresentaram uma porcentagem maior de deglutições com aspirações. Esses pacientes com queixa de disfagia com tumores na faringe e laringe consumiram $<50 \%$ de sua nutrição oralmente e com a consistência e variedade de alimentos limitada, apresentando um número significativamente maior da porcentagem de deglutições com aspirações (Pauloski, et al., 2002).

$\mathrm{O}$ tratamento do CCP pode ser realizado por meio de cirurgia, quimioterapia, radioterapia e a associação entre eles. A QV desses indivíduos está alterada, com isso, é necessário um planejamento de intervenções para amenizar o estresse dos pacientes com câncer, proporcionando uma melhor reabilitação (Sawada, et al., 2006).

De acordo com a literatura, o CCP inclui tumores malignos do trato aerodigestivo superior, que abrange cavidade oral, orofaringe, hipofaringe, nasofaringe e laringe. Fatores de risco importantes para o desenvolvimento do CCP são o tabagismo e etilismo, relacionados com a intensidade e duração do hábito, além da associação entre eles (Alvarenga et al, 2008). Outros fatores de risco associados ao CCP podem ser alimentação pouco balanceada, pobre em vegetais e frutas, fatores ocupacionais relacionados ao trabalho em ambiente externo e exposição a substâncias químicas (Boing \& Antunes, 2011). 
Research, Society and Development, v. 10, n. 9, e0310917630, 2021

(CC BY 4.0) | ISSN 2525-3409 | DOI: http://dx.doi.org/10.33448/rsd-v10i9.17630

No estudo de Aquino, et al. (2016), foram estudados 18 casos de óbitos por câncer de lábio, cavidade oral e orofaringe, $72,2 \%$ realizaram quimioterapia, $66,6 \%$ fizeram radioterapia e 27,8\% sofreram intervenção cirúrgica. Todos os casos estudados apresentaram alterações nas funções estomatognáticas, onde 88,9\% apresentaram alterações na fala, 88,3\% manifestaram alterações na mastigação e deglutição e 72,2\% obtiveram alterações de sucção.

Em casos de câncer de cabeça e pescoço, muitos estudos indicam que é comum ocorrer alterações das funções que envolvem a alimentação, como deglutição, mastigação e sucção, além de alterações da comunicação, sendo necessário um restabelecimento das funções (Aquino, et al., 2016).

A disfagia é um sintoma frequentemente relatado antes do tratamento do câncer, em resultância de anormalidades estruturais ou função dos órgãos envolvidos na deglutição (Pootz, et al., 2020). Podem ocorrer alterações no processo de deglutição em decorrência do próprio tumor, porém a radioterapia é um fator complementar para ocorrência e gravidade da disfagia, devido a danos na musculatura e estruturas (Rebouças et al., 2011).

No estudo de Pauloski, et al. (2002) presente nesta pesquisa, analisando a função da deglutição, pacientes com queixa de dificuldade na deglutição apresentaram menor eficiência para deglutir, maior tempo de trânsito oral e faríngeo e mais goles com aspiração, além de consumir menos da sua nutrição por via oral e evidenciando que não eram capazes de comer todas as consistências, em comparação a aqueles que não apresentaram queixa. Demonstra que os pacientes com câncer de cabeça e pescoço, tratados com radioterapia e quimioterapia, foram capazes de identificar uma piora e dificuldade da sua função da deglutição, como a disfagia.

Pacientes com queixas de disfagia apresentaram uma porcentagem maior de deglutições com aspiração, porém pacientes apresentaram aspiração silente após o tratamento de câncer, o que indica que mesmo que a queixa de disfagia pode ser um indicador de aspiração, porém é possível que pacientes com aspiração silente não percebam a sua dificuldade de deglutição (Pauloski, et al., 2002).

Na pesquisa de Silva, et al. (2009), foram analisados os dados de 178 pacientes com câncer de boca e orofaringe, foram analisados dados de pacientes submetidos a procedimentos, que incluem cirurgia, esvaziamento cervical, radioterapia, quimioterapia, combinação entre eles e nenhum procedimento. Em relação a esses procedimentos, pacientes que não realizaram nenhum procedimento, $35,7 \%$ apresentaram queixa de disfagia, pacientes que realizaram a combinação da radioterapia e quimioterapia, $23,8 \%$ apresentaram queixa de disfagia, radioterapia isolada onde $13,4 \%$ apresentaram queixa e quimioterapia isolada a porcentagem foi de $5,1 \%$ dos pacientes apresentaram queixa. Em relação a pacientes com queixas fonoaudiológicas, as alterações mais presentes em orofaringe foram queixa de disfagia, onde 41,5\% apresentaram a queixa e $26,2 \%$ apresentaram queixa de disfagia associada à otalgia.

O tratamento mais recorrente, dos pacientes com neoplasias em orofaringe, foi a combinação entre radioterapia e quimioterapia e a queixa fonoaudiológica mais recorrente foi a disfagia. No estudo, os pacientes submetidos a radioterapia e quimioterapia possuíam mais queixas de disfagia. Na literatura, o tratamento do câncer de cabeça e pescoço e o próprio tumor podem causar alterações nas estruturas e nas funções do sistema estomatognático, incluindo as alterações de deglutição (Silva, et al, 2009).

Em muitos casos de câncer que atingem a orofaringe, o acompanhamento fonoaudiológico é essencial, orientando e realizando terapia com os pacientes, das consequências do tratamento e da própria doença, que atinge as funções estomatognáticas, como mastigação e deglutição (Oliveira, et al., 2015). O fonoaudiólogo é o profissional recomendado para verificar qual o tratamento ideal e realizar a intervenção adequada, para determinar a consistência alimentar ideal e praticar os exercícios apropriados. (Leonor, et al., 2015).

Segundo o estudo de van den Berg, et al. (2015) presente nesta pesquisa, a terapia de deglutição não reduziu a 
Research, Society and Development, v. 10, n. 9, e0310917630, 2021

(CC BY 4.0) | ISSN 2525-3409 | DOI: http://dx.doi.org/10.33448/rsd-v10i9.17630

gravidade de disfagia, além de não melhorar a alimentação social e o estado nutricional durante o tratamento com radioterapia, porém as compensações e os exercícios de alongamento realizados na terapia de deglutição do estudo visam uma deglutição segura, acelerarando a recuperação da função da deglutição.

No estudo de caso de Sobol, et al. (1979) presente nesta pesquisa, aborda sobre a dificuldade de deglutição, nos casos de câncer de cabeça e pescoço, impede a nutrição oral adequada, onde a desnutrição pode acarretar perda de peso e afetar a imunidade. A necessidade de ingestão de proteína e calorias são determinadas pelo estado nutricional, além disso, existem vários problemas com os métodos de alimentação por tubo, que podem impedir os pacientes a terem uma nutrição enteral adequada. A hiperalimentação intravenosa foi um método rápido e relativamente seguro e eficaz para restaurar o estado nutricional dos pacientes com câncer de cabeça e pescoço.

No estudo de Vieira, et al (2014), foram avaliados o perfil nutricional de 24 pacientes com diagnóstico de câncer de cabeça e pescoço, onde 66,7\% apresentavam desnutrição, sendo 33,3\% classificados como desnutridos graves. Muitos pacientes com câncer de orofaringe tem uma alimentação muito debilitada, em decorrência de diversos fatores, e também possuem uma relevante perda de peso involuntária, fazendo-se essencial, em alguns casos, a terapia nutricional enteral por sonda.

\section{Considerações Finais}

Pacientes, com câncer de cabeça e pescoço, que apresentam queixa de disfagia tem uma piora da função da deglutição, o que limita sua ingestão oral e a percepção da sua função da deglutição pode ser útil no automonitoramento da disfagia durante a terapia de deglutição. Adicionar terapia de deglutição não melhora a normalidade de ingestão de alimentos, mas acelera a recuperação da função da deglutição. Alimentação enteral muitas vezes não atende a demanda dos pacientes com câncer, como o suporte nutricional é fundamental no tratamento do câncer, então a hiperalimentação venosa é uma alternativa viável e eficiente em alguns casos específicos em que a via oral não é possível e há impedimento para a nutrição enteral.

Sugere-se que estudos futuros sejam realizados com maior número amostral e maior homegeneidade relacionada aos tipos de cânceres de orofaringe e seu tratamento. Além disso, sugerem-se maiores estudos que abordem as alterações de deglutição e seu impacto na nutrição nesta população.

\section{Referências}

Alvarenga, L. M., Ruiz, M. T., Pavarino-Bertelli, E. C., Ruback, M. J. J., Maniglia, J. V. \& Goloni-Bertollo, M. (2008). Avaliação epidemiológica de pacientes com câncer de cabeça e pescoço em um hospital universitário do noroeste do estado de São Paulo. Revista Brasileira de Otorrinolaringologia, 74 (1), 68-73.

Aquino, C. A. R., Lima, L. T. M. L., Menezes, X. C. R. C. \& Rodrigues, M. (2016). Alterações fonoaudiológicas e acesso ao fonoaudiólogo nos casos de óbito por câncer de lábio, cavidade oral e orofaringe: um estudo retrospectivo. Revista CEFAC, 18 (3), 737-745.

Boing, A. F. \& Antunes, J. L. F. (2011). Condições socioeconômicas e câncer de cabeça e pescoço: uma revisão sistemática de literatura. Ciência \& Saúde Coletiva, São Paulo, 16 (2), 615-622.

Dedivitis, R. A., França, C. M., Mafra, A. C. B., Guimarães, F. T. \& Guimarães, A. V. (2004). Características clínico-epidemiológicas no carcinoma espinocelular de boca e orofaringe. Revista Brasileira de Otorrinolaringologia, 70 (1), 35-40.

Furkim, A. M. \& Sacco, F. B. A. (2008). Eficácia da fonoterapia em disfagia neurogênica usando a escala funcional de ingestão por via oral (FOIS) como marcador. Revista Cefac, 10 (4), 503-512.

Galbiatti, S. L. A., Padovani-Junior, J. A., Maníglia, J. V., Rodrigues, C. D. S., Pavarino, É. C. \& Goloni-Bertollo, E. M. (2013). Head and neck cancer: causes, prevention and treatment. (2013). Brazilian Journal Of Otorhinolaryngology, 79 (2), 239-247.

Leonor, V. D., Santos, R. S., Mendes, J. M. \& Willig, M. H. (2015). As contribuições da educação continuada em disfagia orofaríngea para a assistência de enfermagem pediátrica em um hospital de ensino. Revista CEFAC, 17 (5), 1531-1540.

Medeiras, F. P. P., Martinez, C. E. \& Cardoso, S. S. (2016). Estado nutricional e ingestão alimentar de pacientes com câncer de cabeça e pescoço submetidos a tratamento oncológico. Arquivos de Ciências da Saúde, 23 (4), 43-47. 
Research, Society and Development, v. 10, n. 9, e0310917630, 2021

(CC BY 4.0) | ISSN 2525-3409 | DOI: http://dx.doi.org/10.33448/rsd-v10i9.17630

Oliveira, F. P., Santos A., Viana, M. S., Alves J. L., Pinho, N. B. \& Reis P. F. (2015). Perfil Nutricional de Pacientes com Câncer de Cavidade Oral em PréTratamento Antineoplásico. Revista Brasileira de Cancerologia, 61 (3), 253-259.

Oliveira, M. M., Malta, D. C, Guauche, H., Moura, L. \& Silva, G. A. E. (2015). Estimativa de pessoas com diagnóstico de câncer no Brasil: dados da Pesquisa Nacional de Saúde, 2013. Revista Brasileira de Epidemiologia, 18 (2), 146-157.

Pauloski, B. R., Rademaker, A. W., Logemann, J. A., Lazarus, C. L., Newman, L., Hamner, A., Maccracken, E., Gaziano, J. \& Stachowiak, L. (2002). Swallow function and perception of dysphagia in patients with head and neck cancer. Head and Neck. 24(6), 555-65.

Pinthon M. M., Sant'Anna L. I. D. A., Baião F. C. S., Santos R. L., Coqueiro R. S. \& Maia L. C. (2015). Assessment of the effectiveness of mouthwashes in reducing cariogenic biofilm in orthodontic patients: a systematic review. Journal of Dentistry, 43 (3), $297-308$.

Pootz, S. C., Boff, D. G., Canuto, R., Brollo, J. \& Silva, A. C. P. (2020). Aconselhamento Nutricional em Pacientes com Câncer de Cabeça, Pescoço e Esôfago em Tratamento (Quimio)Radioterápico. Revista Brasileira de Cancerologia, 665 (1), 01-10.

Rebouças, L. M., Callegaro, E., Gil, G. O. B., Silva, M. L. G., Maia, M. A. C. \& Salvajoli, J. V. (2011). Impacto da nutrição enteral na toxicidade aguda e na continuidade do tratamento dos pacientes com tumores de cabeça e pescoço submetidos a radioterapia com intensidade modulada. Radiologia Brasileira, 44 (1), 42-46.

Sawada, O. N., Dias, A. M. \& Zago M. M. F. (2006). O efeito da radioterapia sobre a qualidade de vida dos pacientes com câncer de cabeça e pescoço. Revista Brasileira de Cancerologia, 52 (4), 323-329.

Shamseer, L., Moher, D., Clarke, M., Ghersi, D., Liberati, A., Petticrew, M., Shekelle, P. \& Stewart, L. A. (2015). Preferred reporting items for systematic review and meta-analysis protocols (PRISMA-P) 2015: elaboration and explanation, BMJ, 349:g7647 doi:10.1136/bmj.g7647

Silva, L. S. P., Leão, L. M. V. \& Scarpel, D. R. (2009). Caracterização da população portadora de câncer de boca e orofaringe atendida no setor de cabeça e pescoço em hospital de referência na cidade de Salvador- BA. Revista CEFAC, 11 (3), 441-447.

Sobol, S. M., Conoyer, J. M \& Sessions, D. G. (1979). Enteral and parenteral nutrition in patients with head and neck cancer. Annals of Otology, Rhinology \& Laryngology, 88 (4 Pt 1), 495-501.

Van Den Berg, M. G., Kalf, J. G., Hendriks, J. C., Takes, R. P., Van Herpen, C. M., Wanten, G. J., Drenth, J. P., Kaanders, J. H. \& Merkx, M. A. (2016). Normalcy of food intake in patients with head and neck cancer supported by combined dietary counseling and swallowing therapy: A randomized clinical trial. Head and Neck, 38 Suppl 1, E198-206.

Vieira, E. M. M., Galvão, A. C. P., Costa, H. C. B. A. L., Amorim, A. C. L., Pinto, J. V., Ribeiro, R. G. S. P. \& Okamoto, A. C. (2014). Perfil nutricional de pacientes oncológicos atendidos no ambulatório de cabeça e pescoço de um hospital filantrópico do município de Cuiabá(MT), Brasil. Archives of Health Investigation, 3 (3), 76-83. 